\title{
The Lorentz transformations by way of the wave equations
}

\author{
Miroslav Pardy \\ Department of Physical Electronics \\ and \\ Laboratory of Plasma physics \\ Masaryk University \\ Kotlářská 2, 61137 Brno, Czech Republic \\ e-mail:pamir@physics.muni.cz
}

October 26, 2020

\begin{abstract}
We prove here, by the rigorous mathematical procedure, that so-called Lorentzian time in the special theory of relativity is defined by the wave equation, where the wave of time is the form of matter and not the Bergson physiological process in $S$ and $S^{\prime}$.
\end{abstract}

Key words. Special theory of relativity, simultaneity, wave equation.

We frequently learn that time in physics is defined by its measurement. Or, time is what a clock reads. In non-relativistic physics, it is a scalar quantity and, like length, mass, and charge, is considered as a fundamental quantity. Time can be combined mathematically with other physical quantities to derive other concepts such as motion, kinetic energy and time-dependent fields. In order to measure time, one can record the number of occurrences (events) of some periodic phenomenon. The sun was the arbiter of the flow of time.

Galileo, Newton, and others up until the 20th century thought that time was the same for everyone everywhere. This is the basis for timelines, where time is a parameter. The modern understanding of time is based on Einstein's theory of relativity, in which rates of time run differently depending on relative motion, and space and time are merged into spacetime. In this view time is a coordinate.

Einstein proved in his well known article and book (Einstein, 1905; 1919; 1922) that the simultaneity in the inertial system $S^{\prime}$ moving with velocity $v$ with regard to the inertial system $S$ is broken. Einstein writes (Einstein, 1905):

So we see that we cannot attach any absolute signification to the concept of simultaneity, but that two events which, viewed from a system of co-ordinates, are simultaneous, can no longer be looked upon as simultaneous events when envisaged from a system which is in motion relatively to that system. 
So, let us write the Lorentz transformation between system $S$ and $S^{\prime}$.

$$
\begin{gathered}
x^{\prime}=\gamma(x-v t), \\
t^{\prime}=\gamma\left(t-\left(v / c^{2}\right) x\right), \\
\gamma=\frac{1}{\sqrt{1-v^{2} / c^{2}}}
\end{gathered}
$$

We get from eq. (2) $t_{1}^{\prime}=\gamma\left(t-\left(v / c^{2}\right) x_{1}\right), t_{2}^{\prime}=\gamma\left(t-\left(v / c^{2}\right) x_{2}\right)$. So, if $x_{1} \neq x_{2}$, then $t_{1}^{\prime} \neq t_{2}^{\prime}$. So, we see that we cannot attach any absolute signification to the concept of simultaneity. The Einstein sofisticated experiment was ignored.

We prove here, by the rigorous mathematical procedure, that so-called Lorentzian time in the special theory of relativity is defined by the wave equation, where the wave is the form of matter and in no case the phlogiston.

We get from equation (2) the following elementary relations with $t^{\prime}=T$

$$
\begin{gathered}
\frac{\partial T}{\partial t}=\gamma=T_{t}, \\
\frac{\partial T}{\partial x}=-\gamma \frac{v}{c^{2}}=T_{x} .
\end{gathered}
$$

After comparison of eq. (4) with eq. (5) we get

$$
\left(\frac{\partial}{\partial t}+\frac{c^{2}}{v} \frac{\partial}{\partial x}\right) T=0
$$

After application of the operator $\left(\frac{\partial}{\partial t}-\frac{c^{2}}{v} \frac{\partial}{\partial x}\right)$ to the equation (6), we get

$$
\left(T_{t t}-\frac{c^{4}}{v^{2}} T_{x x}\right)=0,
$$

which is the partial differential equation describing motion of time as some medium. So, This is the mathematical prove that time is a form of matter.

If we perform the same analogous procedure with the x-transformation, we get the analogous quation with $x^{\prime}=X$. Or,

$$
\left(X_{t t}-v^{2} X_{x x}\right)=0 \text {. }
$$

So, we see that partial differential equatio for $T$ and $X$ is the standard wave equation of the form

$$
\frac{1}{a^{2}} \frac{\partial^{2} u(x, t)}{\partial t^{2}}-\frac{\partial^{2} u(x, t)}{\partial x^{2}}=0 .
$$

If we include the boundary conditions

$$
T(0, t)=0 ; \quad T(l, t)=0,
$$

we can prove that the solution of the wve equation is of the form: 


$$
T=\sum_{k=1}^{\infty}\left(a_{k} \cos \frac{k \pi a t}{l}+b_{k} \sin \frac{k \pi a t}{l}\right) \sin \frac{k \pi x}{l} .
$$

We can say that if the boundary conditions (10) are with the physical meaning, then the solution (11) is also of the physical meaning. Such statement is in agreement with the Wigner approach to physics because he speaks on the unreasonable effectivenses of mathematics in the natural sciences (Wigner, 1960).

The vector form of eqs. (1) and (2) is as follows (Fok, 2015):

$$
\begin{gathered}
\mathbf{x}^{\prime}=\mathbf{x}-\mathbf{v} t+(\gamma-1) \frac{\mathbf{v}}{v^{2}}(\mathbf{v} \cdot \mathbf{x}-\mathbf{v} t) \\
t^{\prime}=\gamma\left(t-\frac{1}{c^{2}}(\mathbf{v} \cdot \mathbf{x})\right)
\end{gathered}
$$

If we apply the analogous procedure with $\mathrm{x}$ - and $\mathrm{t}$-transformation to $\mathrm{x}, t$, we get

$$
\begin{gathered}
\left(\frac{1}{v^{2}} \frac{\partial^{2}}{\partial t^{2}}-\Delta\right) \mathbf{X}=0 \\
\left(\frac{v^{2}}{c^{4}} \frac{\partial^{2}}{\partial t^{2}}-\Delta\right) T=0 \\
\gamma=\frac{1}{\sqrt{1-v^{2} / c^{2}}} .
\end{gathered}
$$

Our statements are in no case paradoxial and they are not involved in the collection of paradoxes of relativity (Goldblatt, 1972; Terletzkii, 1966) and in the relativistic paradoxes in American Journal of Physics. To our knowledge, our results (LorentzPardy equations (7-8) and (14-15) are not involved in any monograph of the relativity theory (Reichenbach, 1958; Mermin, 1968; Savitt, 2011). Nevertheless, the thinking of the relation of our results to the author results in the scientific physical journal (Pardy, 1969) is very useful.

There is no doubt that the discussion based on our wave equations for time and space will refresh the interest in the theory of relativity described by the Lorentz-Pardy equations of mathematical physics.

\section{References.}

Einstein, A. (1905). Zur Elektrodynamik bewegter Körper, Annalen der Physik, 17. See also The Principle of Relativity, published in 1923 by Methuen and Company, Ltd. of London. Most of the papers in that collection are English translations by W. Perrett and G.B. Jeffery from the German Das Relatitivitätsprinzip, 4th ed., published in 1922 by Teubner.

Einstein, A. (1919). Über die spezielle und die allgemeine relativitäts Theorie, Vierte Auflage, Vieweg \& Sohn, Braunschweig.; chapter 9.

Fock, V. (2015). The Theory of Space, Time and Gravitation, Pergamon; 2nd Edition 
Goldenblat, I. I. The time paradoxes in the special theory of relativity, (NAUKA, Moscow, 1972), (in Russian).

Mermin, N. D. Space and Time in Special Relativity (McGrawHill, 1968).

Pardy, M. (1969). A remark on the clock paradox, Phys. Lett. 28 A, No. 11, 766

Reichenbach, H. The Philosophy of Space and Time (Dover Publications, Inc., 1958).

Savitt, S. Time in the Special Theory of Relativity The Oxford Handbook of Philosophy of Time (Oxford, University Press, 2011), Edited by Craig Callende.

Terletzkii, Ya. P. The paradoxes of the special theory of relativity, (NAUKA, Moscow, 1966), (in Russian).

Wigner, E. The Unreasonable Effectivenses of Mathematics in the Natural Sciences, Reprinted from Communications in Pure and Applied Mathematics, Vol. 13, No. I. New York: John Wiley \& Sons, Inc. Copyright - 1960 by John Wiley \& Sons, Inc. 